\title{
DOE Based High-Performance Gate-Level Pipelines
}

\author{
Juan Núñez, María J. Avedillo and Héctor J. Quintero \\ Instituto de Microelectrónica de Sevilla, IMSE-CNM (CSIC/Universidad de Sevilla) \\ Av. Américo Vespucio s/n 41092, Seville (Spain) \\ \{jnunez,avedillo,quintero\}@imse-cnm.csic.es
}

\begin{abstract}
Domino dynamic circuits are widely used in critical parts of high performance systems. In this paper we show that in addition to the functional limitation associated to the noninverting behavior of domino gates, there are also performance disadvantages when compared to inverting dynamic gates, which can be related to this feature. These penalties rise from the fact that in order to produce a logic one, a non-inverting gate requires one or more of its inputs to be also at logic one. We analyze the operation of gate-level pipelines implemented with domino and with Delayed Output Evaluation (DOE), an inverting dynamic gate we have recently proposed, and compare their performance. Using domino and DOE gates similar in terms of delay, improvements in operating frequencies around $50 \%$ have been obtained by the DOE pipelines.
\end{abstract}

Keywords- Nanopipeline, Dynamic logic, Robust design techniques.

\section{INTRODUCTION}

Design of functional units implementing very fine-grained pipelining for high performance applications is currently an area of active research. These solutions do not apply conventional pipeline techniques which insert flip-flops to short down signal propagation paths in combinational logic, but instead rely on logic circuit styles which naturally exhibit the capacity to block data propagation, and thus, are well suited to implement pipeline architectures without memory elements. Potential of dynamic logic, with its precharge and evaluation phases, to implement this kind of pipelining was long ago recognized. Thus, in [1] the operation of the wellknown dynamic-based domino logic in a pipelined fashion using an overlapping multi-phase clock scheme and without latches between consecutive clock phases (superpipeline) was analyzed in depth. It is known that many variations of this multi-phase solution have been developed achieving high performance [2]-[8] and some of them have been applied to speed up critical parts of commercial microprocessors. In particular, architectures with a single gate per clock phase (nanopipeline) have been proposed and demonstrated large operating frequency and throughput [4],[5],[7],[8]. In this context, development of novel domino-based topologies for dynamic gates exhibiting good performance-robustness tradeoff and/or tolerance to process variations is an area of active research [9],[10],[11].

In spite of their speed advantages, it is well known that domino-based gates exhibit limitation such that only noninverting blocks can be chained (a static inverter is added between each two dynamic stages to guarantee that all inputs to the next logic block are set to 0 after the pre-charge period).
In addition to the functional limitation associated to the noninverting behavior of domino gates, we claim that there are also speed limitations in pipelined networks which can be related to this feature. These penalties rise from the fact that in order to produce a logic one, a non-inverting gate requires one or more of its inputs to be also at logic one. This translates in that logic ones can degrade as they propagate through the logic network eventually leading to a functional failure.

Recently we have proposed an inverting dynamic gate topology called Delayed Output Evaluation (DOE) [12]. DOE exhibits good speed - noise tolerance tradeoffs which are attractive for DSM technologies. In addition, we have identified that its inverting nature allows operating frequency improvements over domino-based style. In this paper we focus on these advantages at the interconnection level. In particular, we analyze the operation of DOE based nanopipelines and compare its performance to domino counterparts.

The paper is organized as follows: in Section 2, the DOE logic style is described and the implications of the noninverting behavior of domino on circuit operation are illustrated. In Section 3, DOE and domino nanopipelines are evaluated through simulations experiments and compared. Finally, some conclusions are given in Section 4.

\section{ARCHITECTURE ANALYSIS}

\section{A. DOE Topology}

Figure 1a shows a generic conventional dynamic gate or domino gate. It operates in two phases called precharge (CLK $=0$ ) and evaluation (CLK = 1). It is composed of a dynamic stage and a static output stage. The dynamic stage realizes the logic functionality while the output stage is required to solve cascading of gates and to drive fan-out. Keeper transistor is added to protect dynamic node against leakage/noise.

Figure 1b shows the schematic of the Delayed Output Evaluation (DOE) topology for a generic gate. Note that the static inverter is changed into a NAND gate whose inputs are the dynamic node and a delayed clock, $V_{C L K}^{D}$, plus a static inverter. Clocks $V_{C L K}$ and $V_{C L K}^{D}$ are also depicted. The rising edge of $V_{C L K}^{D}$ is delayed with respect to the rising edge of $V_{C L K}$ by $\Delta_{\mathrm{CLK}}$, while, ideally, both falling edges are simultaneous. For $V_{C L K}^{D}=0, V_{N A N D}$ is pulled up independently of $V_{D Y N}$. The static inverter is added guarantying that the precharge value of the gate output $\left(V_{\text {OUT }}\right)$ is low as in domino logic. For $V_{C L K}^{D}=1$, 


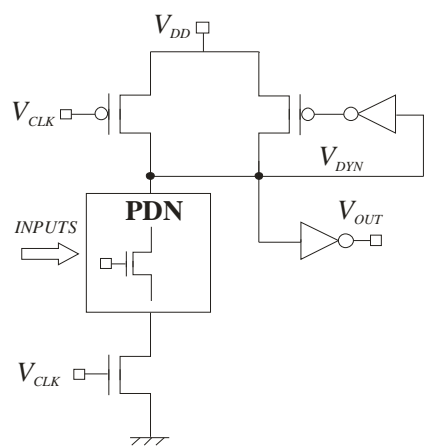

(a)

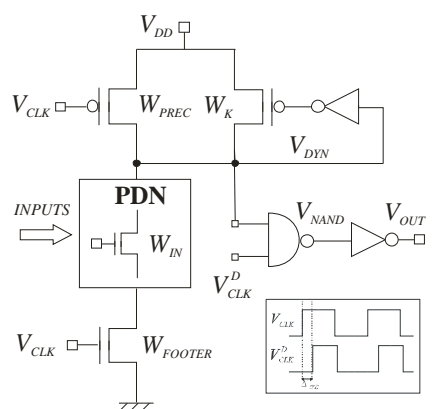

(b)

Fig 1. (a) Domino gate. (b) DOE gate.

the NAND gate evaluates its input. For those input combinations which discharge the dynamic node, the pulldown network is off and gate output remains low. For input combinations which do not discharge dynamic node, the NAND output node is pulled down and $V_{\text {OUT }}$ is pulled up.

The evaluation delay in DOE gate is determined by the speed of the NAND-INV static stage and by the amount by which evaluation of the NAND is delayed. Gate delay is to some extend independent of how fast dynamic node discharges. As a result, achieved delay-noise tolerance tradeoff is significantly better than in domino gates as we showed in [12]. Also, the design of a Kogge-Stone adder using DOE gates is reported in [12] in order to validate their capability to build up logic networks.

However this is not the only advantage of the DOE topology. As already mentioned when describing its operation, DOE produces a logic one when an input combination which does not discharge the dynamic node is applied. Clearly, this is not the behavior of the domino gate. It is the result of adding an inverting stage to the domino gate. Next sub-section illustrates how this translates in speed and robustness improvements when interconnecting gates.

\section{B. Interconnecting gates}

We compare a chain of ten 16-input NOR DOE gates with a chain of 16-input OR domino gates. Both circuits are operated in a gate-level pipeline with a three-phase overlapped clock scheme as depicts in Fig. 2a. Gates are connected such that input changes propagate through the circuit and each gate is excited with the worst case input combination. A sequence alternating "0" and " 1 " is applied. Dynamic stages as well as

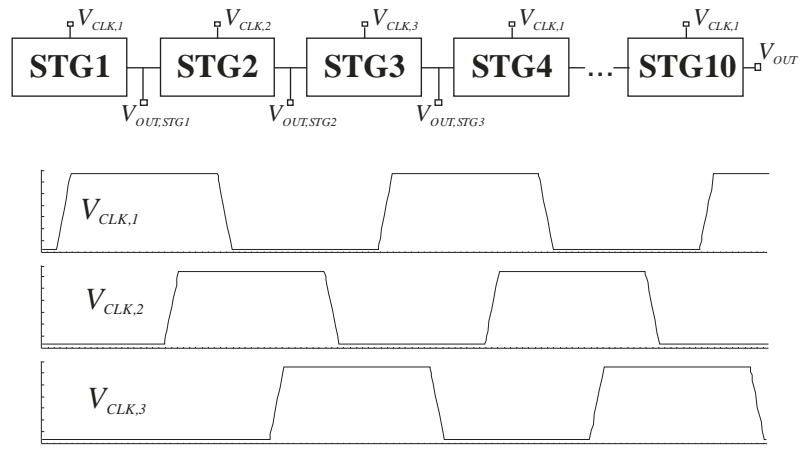

(a)

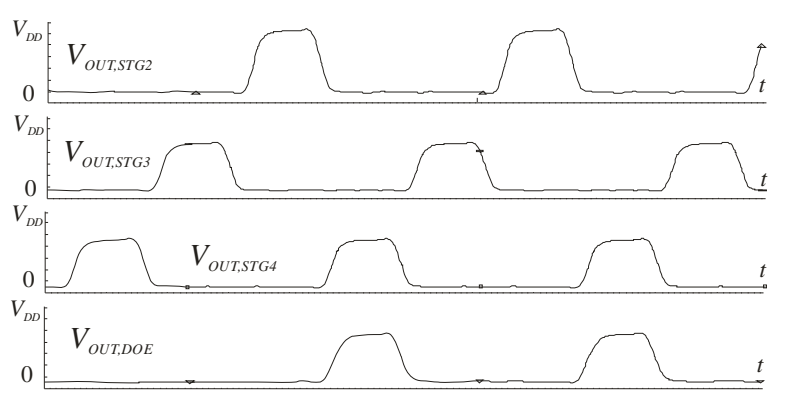

(b)

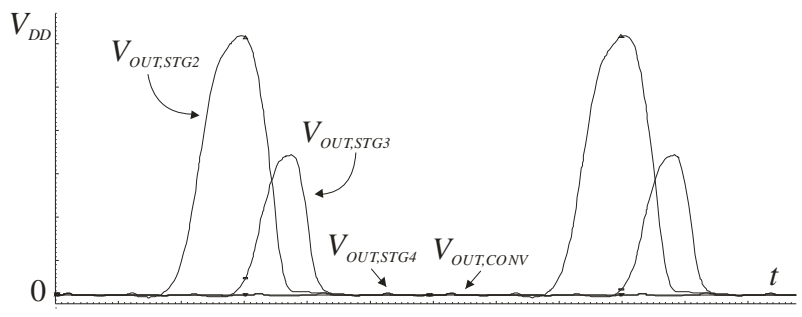

(c)

Fig 2. (a) Three-phases clock scheme. (b) Simulation results corresponding to a chain of ten 16-inputs DOE NOR gates. (c) Simulation results of its conventional domino counterpart.

keeper transistor and feedback and output inverters have been identically sized in both circuits and such that the delays of both gates are equal. However, maximum operating frequency of DOE network is higher than its domino counterpart. We have analyzed in depth the operation of both circuits.

Figure 2 depicts results for both simulated circuits. DOE circuit (Fig. 2b) produces correct output at the simulated frequency $\left(V_{\text {OUT,DOE }}\right)$. Domino (Fig. 2c) does not work $\left(V_{\text {OUT,CONV }}\right)$. The outputs of intermediate stages for both CONV and DOE are also shown. It can be observed how domino outputs degrade with the number of stages but DOE does not.

The differences could be explained on the basis of the input combination producing a zero-to-one transition of the gate output for each topology. In domino, being non-inverting, this output transition is associated with inputs combinations discharging the dynamic node. Discharging of the dynamic node requires one or more inputs being at logic one. "Good" 


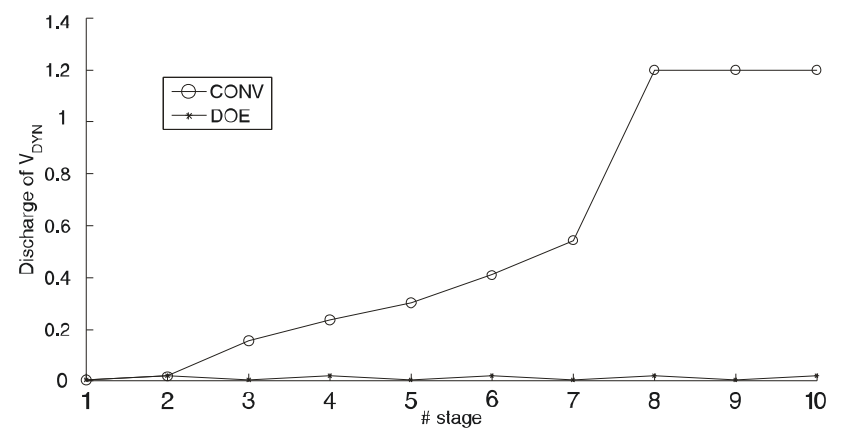

Fig 3. Voltage level to which $V_{D Y N}$ of each stage discharges for both topologies.

ones are required to fully discharge dynamic node and produce a "good" output one. Otherwise, functional failures can occur after several stages. Contrary, in DOE, implementing inverting functionality, the zero to one output transition occurs for input combinations which do not discharge dynamic node. Thus, how good the output logic one is does not depend on how good the input logic ones are. Unlike domino, degraded logic ones do not propagate through the circuit, and so do not accumulate, leading to functional failures after several stages.

It is interesting to analyze the behavior of the dynamic node in each circuit. For that, the voltage level to which each dynamic node discharges has been measured. Figure 3 depicts this voltage level versus stage number for domino (at $V_{D D}$ $=1.2 \mathrm{~V}$ ) and DOE (at $V_{D D}=1.2 \mathrm{~V}$ and $V_{D D}=1 \mathrm{~V}$ ) networks. Voltage levels are identical for stage number one. It can be observed that in domino, the discharge of the dynamic node degrades in consecutive stages and the complete chain does not operate correctly. DOE behavior is completely different. Minimum voltage level is slightly increased from first stage to second one due to non-ideal inputs but then remains constant, even for the lower $V_{D D}$ value.

\section{EXPERIMENTAL RESULTS}

Experiments have been carried out in order to complete the comparison between domino and DOE pipelines operation. Different ten stage chains like those described in previous section have been simulated. In all the simulated chains NOR (OR) gates are used for DOE (domino) circuits. Five different chain -pairs are characterized in a commercial $1.2 \mathrm{~V} 130 \mathrm{~nm}$ technology. They differ only in the size of the keeper transistor. Table I reports gate delays for some of the keeper sizes. Keeper width increases from gate $\mathrm{K} 1$ to gate $\mathrm{K} 2$. Larger keeper transistor widths imply that the dynamic node discharges more slowly. As expected, domino delay increases while DOE delay keeps constant, although discharging rate of dynamic node does impact the operation of the DOE pipelines as well. DOE and domino delays are similar for gate version $\mathrm{K} 3$. Both in domino and in DOE, noise tolerance increases upsizing keeper transistor.
TABLE I. GATE DELAYS

\begin{tabular}{|c|c|c|}
\hline \multirow{2}{*}{} & \multicolumn{2}{|c|}{ Delay (ps) } \\
\cline { 2 - 3 } & Domino & DOE \\
\hline K1 & 71.9 & 86.1 \\
\hline K3 & 89.8 & 86.4 \\
\hline K5 & 108.1 & 86.6 \\
\hline
\end{tabular}

Delayed clock required for DOE operation is generated inside each gate by means of a pair of inverters.

Different frequencies have been measured to characterize the operation of each working (correct behavior) pipelined chain. These frequencies are:

- F1: Clock frequency up to which every gate behaves "ideally". By "ideally" we mean that dynamic nodes are fully discharged and charged in worst case scenarios and "1" inputs to every stage are ready (90\% of final value reached) when evaluation clock rises.

- F2: Clock frequency up to which every dynamic node discharges under $100 \mathrm{mV}$ and charges over $1.1 \mathrm{~V}$.

- F3: Maximum clock operating frequency. Correct output is obtained.

- F4: Clock frequency up to which correct behavior is obtained in SS, FF, SF and FS corners. Supply voltage is reduced by $10 \%$ in $\mathrm{SS}, \mathrm{SF}$ and $\mathrm{FS}$ corners and increased by same amount in FF corner.

- F5: Clock frequency up to which a correct operation is observed for a $30 \mathrm{MC}$ simulations $(3-\sigma)$.

Table II summarizes obtained results. Frequencies have been normalized with respect to the smallest one measured in the experiment (F4 for domino K5).

TABLE II. CHARACTERIZATION OF THE NORMALIZED FREQUENCIES OF THE PIPELINES

\begin{tabular}{|c|c|c|c|c|c|c|}
\hline \multirow{2}{*}{} & \multicolumn{2}{|c|}{$\boldsymbol{F 1}$} & \multicolumn{2}{c|}{$\boldsymbol{F 2}$} & \multicolumn{2}{c|}{$\boldsymbol{F 3}$} \\
\cline { 2 - 7 } & Dom. & DOE & Dom. & DOE & Dom. & DOE \\
\hline K1 & 3.13 & 2.50 & 3.31 & 3.75 & 3.25 & 4.06 \\
\hline K2 & 2.69 & 2.50 & 2.94 & 3.50 & & \\
\hline K3 & 2.25 & 2.50 & 2.63 & 3.38 & 2.63 & 3.81 \\
\hline K4 & 1.75 & 2.50 & 2.13 & 3.19 & & \\
\hline K5 & 1.38 & 2.50 & 1.63 & 3.00 & 1.88 & 3.50 \\
\hline
\end{tabular}

\begin{tabular}{|c|c|c|c|c|}
\hline \multirow{2}{*}{} & \multicolumn{2}{|c|}{$\boldsymbol{F 4}$} & \multicolumn{2}{c|}{$\boldsymbol{F 5}$} \\
\cline { 2 - 5 } & Dom. & DOE & Dom. & DOE \\
\hline K1 & 2.00 & 2.56 & 3.06 & 3.19 \\
\hline K2 & & & 2.63 & 3.06 \\
\hline K3 & 1.56 & 2.38 & 2.13 & 3.00 \\
\hline K4 & & & 1.81 & 2.88 \\
\hline K5 & 1.00 & 2.00 & 1.44 & 2.75 \\
\hline
\end{tabular}


It is observed that F1 values are independent of the keeper size in DOE circuits. In these circuits, frequency F1 is limited by the criteria associated to the readability of inputs in all the cases and so, since gate delay is the same for all gate versions, so it is $\mathrm{F} 1$. In domino $\mathrm{F} 1$ decreases from $\mathrm{K} 1$ to $\mathrm{K} 5$ as expected since gate delays increase. It is interesting to compare $\mathrm{K} 3$ results as both gate delays and dynamic node behavior are similar for domino and DOE in this case. Note that DOE F1 is slightly higher than in domino $(\sim 10 \%)$. This is due to the fact that although evaluation delays (reported in Table 1) are similar, precharge delays are not. They are larger in the DOE gates and it translates in small advantage for pipeline operation. However these advantages are limited. We expect larger differences on the basis of the distinct behaviors pointed out in previous section.

Concerning F2, it can be observed that the operating frequency of the DOE chain is higher than the operating frequency of the domino chain for all the keeper transistors. Even for those for which the delay of the domino gate is smaller than the delay of its DOE counterpart (K1 and K2). Improvements increase from $\mathrm{K} 1$ to $\mathrm{K} 5$ as expected, ranging from $13 \%$ to $85 \%$. As we anticipated, larger differences between domino and DOE are observed. For example, for K3, which corresponds to almost equal gate delays, improvement rises from $11 \%$ (when comparing $\mathrm{F} 1$ ) to $29 \%$ (F2). In addition to the advantages associated to the larger precharge times previously pointed out, there are advantages which rise from the inverting feature of the DOE gates discussed in Section 2. Since discharging of dynamic node does not degrade through the chain, the operating frequencies fulfilling the F2 criteria are higher.

Also F3 of DOE is higher than the F3 of the domino chains for all the keeper transistors. Improvements range from $25 \%$ (K1) to $87 \%$ (K5). For K3, improvement rises from $29 \%$ (when comparing F2) to $45 \%$ (F3). No differences are observed between F2 and F3 improvements for K5. This probably is the result of a bad DOE design for the largest keeper K5.

Concerning F4, improvements range from $28 \%$ (K1) to $100 \%$ (K5). It is interesting to compare the results obtained for F1 (very conservative design criteria) and for F4. For example, for $\mathrm{K} 3, \mathrm{~F} 4$ is $30 \%$ smaller than $\mathrm{F} 1$ for domino, but only $5 \%$ smaller for DOE. These results show the better performance including variability robustness of DOE with respect to domino. Other experiments have been carried out in order to analyze in depth the performance of both architectures.

Monte Carlo analysis has been applied. Clock skew/jitter simulation has also been considered in this experiment. For these random variables with Gaussian distributions have been associated to the position of the edges of the three clock phase signals. The frequency up to which correct operation is observed in $30 \mathrm{MC}$ simulations has been measured. This frequency is reported as F5 in Table II. Better performance of DOE is clearly observed.

Analyzing results for $\mathrm{K} 3$ is relevant since, as we have already repeatedly pointed out, DOE and domino gate delays are similar. For this gate version, DOE is better than domino for all measured frequencies. Achieved improvement goes from $10 \%$ for $\mathrm{F} 1$ to $52 \%$ for $\mathrm{F} 4$. Even higher differences can be obtained in cases for which noise tolerance constraints would require keeper transistor sizes for which DOE gate Delays are smaller than domino ones.

\section{CONCLUSIONS}

We have analyzed the operation of gate-level pipelines implemented with domino and with DOE and their performance in terms of operating frequency. The differences are explained on the basis of the input combination producing a zero to one transition of the gate output for each topology. In domino, being non-inverting, a non-ideal one degrades as it propagates through the logic network eventually leading to a functional failure. Unlike domino, degraded logic ones do not propagate in DOE circuit. Using domino and DOE gates similar in terms of delay, improvements in operating frequencies around 50\% have been obtained for ten-stages chains of high-fan in gates operated in a gate-level pipelined architecture with three clock phases.

\section{ACKNOWLEDGMENT}

This work has been funded by Ministerio de Economía y Competitividad del Gobierno de España with support from FEDER under Project TEC2010-18937 and TEC2011-28302.

\section{REFERENCES}

[1] D. Harris and M.A. Horowitz, "Skew-tolerant domino circuits", IEEE Journal of Solid-State Circuits, vol.32, no.11, pp.1702-1711, Nov. 1997.

[2] R. Hossain, "High Performance ASIC Design", Cambridge, 2008.

[3] S. Horne, D. Glowka, S. McMahon, P. Nixon, M. Seningen and G. Vijayan, "Fast14 Technology: design technology for the automation of multi-gigahertz digital logic", International Conference on Integrated Circuit Design and Technology, pp. 165- 173, 2004

[4] W. Belluomini; D. Jamsek; A. Martin; C. McDowell; R. Montoye; T. et al. "An $8 \mathrm{GHz}$ floating point multiply", IEEE International Solid-State Circuits Conference, pp. 374-604., 2005.

[5] J. Sivagnaname, H.C. Ngo, K.J. Nowka, R.K. Montoye and R.B. Brown,"Wide limited switch dynamic logic circuit implementations", IEEE International Conference on VLSI Design, 2006.

[6] R.J. Sung and D.G. Elliot, "Clock-logic domino circuits for high-speed and energy-efficient microprocessor pipelines", IEEE Transactions on Circuits and Systems II: Express Briefs, vol. 54, no.5, pp. 460-464, 2007.

[7] C.K. Jerry, W.-H. Ma, S. Kim and M. Papaefthymiou, "2.07 GHz floating-point unit with resonant-clock precharge logic", IEEE Asian Solid State Circuits Conference (A-SSCC), pp.1-4, Nov. 2010.

[8] Z. Owda, Y. Tsiatoushas and T. Haniotakis, "High Performance and Low Power Dynamic Circuit Design" IEEE New Circuits and System Conference pp. 502-505, 2011.

[9] A. Peiravi and M. Asyaei, "Current-Comparison-based Domino: new low-leakage high speed domino circuit for wide fan-in gates", IEEE Trans. On Very Large Scale Integration Systems. no. 21, vol. 5, pp.934943, 2012.

[10] A. Alvandpour, R.K. Krishnamurthy, K. Soumyanath and Shekhar Y. Borkar. A su-130-nm condicional Keeper technique. IEEE Journal of Solid-State Circuits. no.37, vol. 5, pp 633-638, May 2002.

[11] H. F. Dadgour and K. Banerjee. A novel variation-tolerant keeper architecture for high-performance low-power wide fan-in dynamic or gates. IEEE Trans. On Very Large Scale Integration Systems. no. 18 vol.11, pp. 1567-1577, 2010.

[12] J. Núñez, M.J. Avedillo, J. M. Quintana, H. J. Quintero. "Novel Dynamic Gate Topology for Superpipelines in DSM Technologies" Proceedings Digital System Design 2013. pp. 280-28, 2013. 